\title{
THE COMPLEX FUZZY CONTROL
}

\section{Enhancing tractor automatic steering precision}

\author{
Song Zhenghe, Lü Antao, Mao Enrong \\ College of Engineering, China Agricultural University, Beijing 100083, P.R. China
}

\begin{abstract}
This paper mostly introduces that it is necessary to apply autonomous tractor in agriculture and how to select control parameter, tractor automatic steering dynamic model is set up and a complex fuzzy control technique is put forward. In addition, fuzzy control formula is designed, which is evaluated by the simulink software. The simulating result reveals that the complex fuzzy control technique produces the behaves similar to the tractor driver, and, it is suitable for tractor automatic steering system.
\end{abstract}

Key words: tractor; autonomous driving; fuzzy control; PID

\section{INTRODUCTION}

Autonomous tractors are believed to be one of the driving forces for precision farming, since they can perform farming operations accurately and automatically. At the same time, it can assure the precision task, such as holding space of ridge in a field. In addition, it can take from the technique request of operator, decrease the overlap operation, and accelerate the rate of progress and economize cost. Document [1] indicates proficiency tractor driver can plough in $10 \mathrm{~cm}$ precision, but after a day's work, precision will recede much more. Whereas, autonomous tractors based on GPS, which cost is $42 \%$ more than general tractors', can maintain operation in $3 \mathrm{~cm}$ precision all time, avoid overlap operation, save fuel consume and cut down dosage of pesticide. So, they can enhance $40 \%$ profit of farm. The study about 
autonomous tractors have been reached in the worldwide, and the research keystone of it is autonomous steering, namely yaw control of the tractor.

Navigation control, which will firstly be designed based on control point, in according to operating mode, is sticking point of autonomous tractor. For example, it is selected in front when tractor used in reaping which dead stocks are equipped, it is selected in rear when tractor used in plough which dead stocks are equipped. Front control point that may be select at front axes and rear control point may be select at rear axes herein putting up vehicle dynamic model. Although there are many navigation control methods, control parameters are all yaw error and yaw angle error, which are limited in permit bound in order that autonomous tractor runs along desire route ${ }^{[2,3]}$. At the same time, data fuse that can make two parameters change into one parameter reinforces data nicety, and the last navigation control is confirmed. In the context vehicle dynamic model based on center of mass is putted up, and a sort of complex fuzzy control technique of tractor automatic steering is put forward, which suits for autonomous tractors.

\section{MODEL OF TRACTOR DYNAMICS}

There are 6 free degrees when tractor runs, H-P Willumeit, who assumed that vehicle was running on a level frictional surface but including vehicle run in plane and tyre sideslip angle, suppose 4 items [4]:

(1) There are 3 free degrees without uprightness movement, pitching movement roll $\mathrm{y}$ shaft and list movement roll $\mathrm{x}$ shaft;

(2) The two wheel on each axle collapse into a single wheel located at the center of the axle, axis load of front and rear only lie in center of axis;

(3) Front wheel sideslip angle, which change is small, and it is directly input losing sight of steer system infection, so model is linearity.

(4) Side force of COM (in this article, COM means the center of mass) equal side force of wheel which lead to wheel side departure, namely angle of wheel plane and wheel onward, and the tyre characteristic change is neglected.

So, a bicycle model was used to describe the tractor dynamic responses, the bicycle model of the tractor should be defined in field coordinates (Fig.1). 


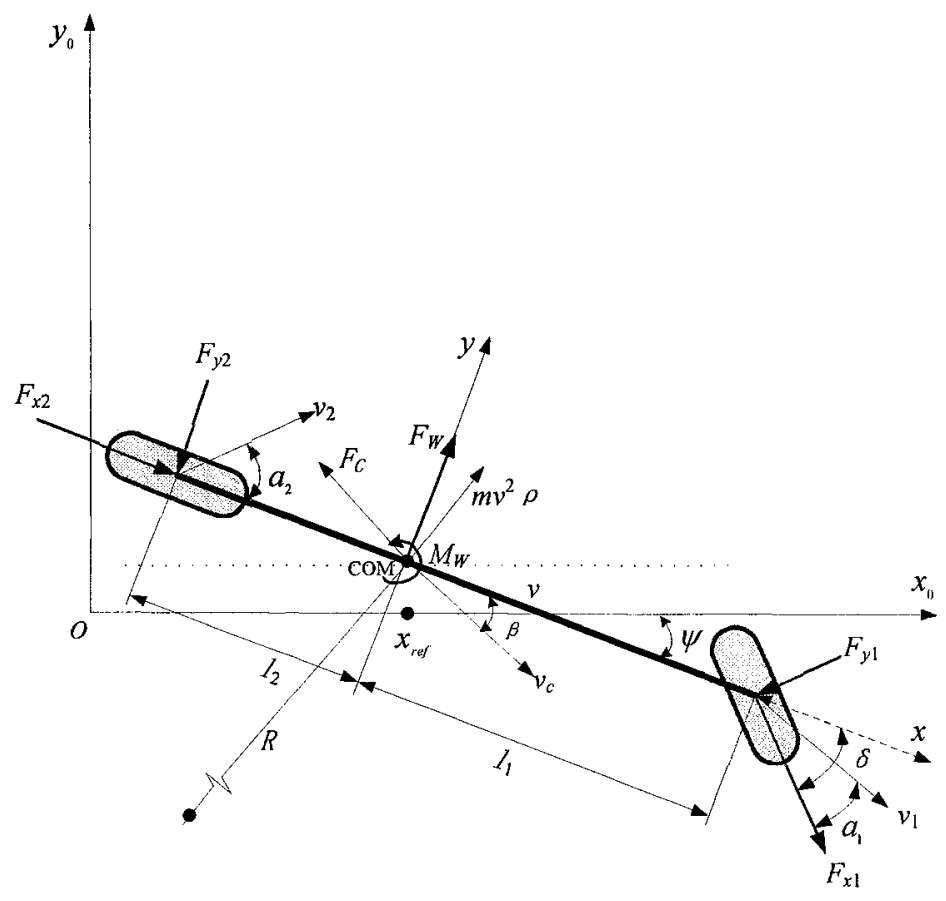

Figure 1. Bicycle model of tractor in field coordinates and tractor-body coordinates

Dynamics equation of the bicycle model about slip angle of tractor and tractor yaw angular acceleration can be defined by the following equations without considering the influence of the side wind:

$$
\begin{aligned}
& \dot{\beta}=A_{11} \beta+A_{12} \dot{\psi}+B_{1} \delta \\
& \ddot{\psi}=A_{21} \beta+A_{22} \dot{\psi}+B_{2} \delta
\end{aligned}
$$

Where, $\quad A_{11}=-\frac{k_{1}+k_{2}}{m v_{c}} ; A_{12}=-1+\frac{k_{2} l_{2}-k_{1} l_{1}}{m v_{c}^{2}} ; \quad A_{21}=\frac{k_{2} l_{2}-k_{1} l_{1}}{M}$; $A_{22}=-\frac{k_{2} l_{2}{ }^{2}+k_{1} l_{1}^{2}}{M v_{c}} ; B_{1}=\frac{k_{1}-F_{x 1}}{m v_{c}} ; B_{2}=\frac{l_{1}\left(k_{1}-F_{x 1}\right)}{M} ; m$ is the mass of tractor, kg; $M$ is the turning inertia with regard to tractor COM, $\mathrm{kg} \cdot \mathrm{m}^{2} ; l_{1}$ and $l_{2}$ are the distance of the front and rear wheel to tractor COM respectively, $\mathrm{m} ; v_{c}$ is the velocity of tractor-body, $\mathrm{km} / \mathrm{h} ; F_{x 1}$ and $F_{x 2}$ are the resistance of the front and rear wheel respectively, $\mathrm{N} ; F_{y 1}$ and $F_{y 2}$ are the side forces at the front and rear wheel respectively, $\mathrm{N} ; F_{W}$ is the side force of side wind, $\mathrm{N} ; M_{W}$ is the moment of side wind, $\mathrm{N} \cdot \mathrm{m} ; \rho$ is the curvature of tractor steering, $\mathrm{m}^{-1}$, 
$R=\frac{1}{\rho} ; \psi$ is the yaw angle, $\mathrm{rad} ; \beta$ is the slip angle of tractor, $\operatorname{rad} ; \delta$ is the steering angle of tractor, rad.

Because lateral speeds of the tractor play important roles in calculating yaw error (distance between COM and $x_{\text {ref }}$ ), the speed can be determined using the following equations:

$$
\dot{y}=v_{c} \sin (\beta+\psi) \approx v(\beta+\psi)
$$

If steering machine is predigested by first-order inertia system, the tractor yaw angle was chosen as a state variable in order to compare it for emulation. The tractor dynamics is then represented in matrix form to determine the slip angle, the yaw rate, the steer angle and the yaw angle of the tractor as following:

$$
\left[\begin{array}{c}
\dot{\beta} \\
\dot{\omega} \\
\dot{y} \\
\dot{\psi} \\
\dot{\delta}
\end{array}\right]=\left[\begin{array}{ccccc}
A_{11} & A_{12} & 0 & 0 & B_{1} \\
A_{21} & A_{22} & 0 & 0 & B_{2} \\
v & 0 & 0 & v & 0 \\
0 & 1 & 0 & 0 & 0 \\
0 & 0 & 0 & 0 & -\frac{1}{\tau}
\end{array}\right]\left[\begin{array}{c}
\beta \\
\omega \\
y \\
\psi \\
\delta
\end{array}\right]+\left[\begin{array}{c}
0 \\
0 \\
0 \\
0 \\
\frac{1}{\tau}
\end{array}\right] \delta_{d}
$$

Where, $\tau$ is the inertia time constant of the steering system; $\delta_{d}$ is the expectation steering angle of the tractor.

\section{YAW COMPLEX FUZZY CONTROL}

Since the action of the tractor wheel is complex in allusion to characteristic of tractor tasks: low speed and no way, and a precision tractor model is not mostly putted up, besides speed change of tractor-body impact the frequency region response, tractor navigation control will avoid depending on tractor dynamics model. However, expert knowledge can be expressed by the fuzzy rule, which can judge state of controlled object real time without precision math model. And, fuzzy control has the characteristic of robust and applicability spontaneously. In this paper, the fuzzy control is introduced into the yaw control of the tractor. PID control is in common used in the industry field, about $84 \%$, which not depends on model and includes proportion, integral and differential coefficient. 
There is strongpoint and shortcoming for PID control and fuzzy control separately. For example, PID control is simple but no good adaptability, the fuzzy control is good adaptability but no control effect when language variable is small. Complex fuzzy control, which fits PID control and fuzzy control together (Fig 2), has strong control function, its principle is that when language variable is small than zero section, fuzzy is disconnection, PID work alone, in order to enhance system stabilization performance.

Complex fuzzy control

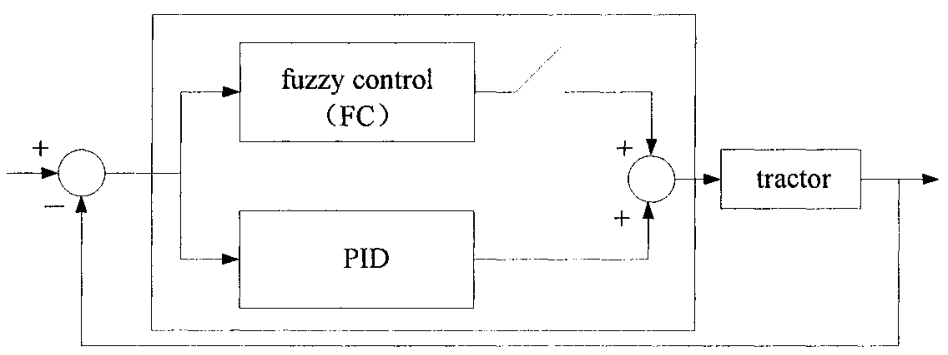

Figure2. Complex fuzzy control system

In this research, a fuzzy controller is selected, which there are two input, yaw error $(y)$ and yaw angle $(\psi)$, and one output $(\delta), y \in\left[\begin{array}{ll}-1 & 1\end{array}\right]$ and $y=\{N B$ NM NS O PS PM PB $\} ; \psi \in[-1.57$ 1.57] and $\psi=\{N B N M N S 0$ $P S P M P B\} ; \delta \in[-0.52330 .5233]$ and $\delta=\{N B N M N S O P S P M P B\}$; Mamdani rule is used, subjection function of language variable is trapezium and triangle, then control rule is showed in table 1, and relation between input and output is show in figure 3.

Table 1. Fuzzy control rule

\begin{tabular}{|c|c|c|c|c|c|c|c|}
\hline & $N B$ & $N M$ & $N S$ & $O$ & $P S$ & $P M$ & $P B$ \\
\hline$N B$ & $N B$ & $N B$ & $N B$ & $N B$ & $N B$ & $N B$ & $N B$ \\
\hline$N M$ & $N B$ & $N B$ & $N B$ & $N B$ & $N B$ & $N B$ & $N B$ \\
\hline$N S$ & $N B$ & $N B$ & $N M$ & $N M$ & $N M$ & $N S$ & $N S$ \\
\hline$O$ & $N B$ & $N M$ & $N S$ & $O$ & $P S$ & $P M$ & $P B$ \\
\hline$P S$ & $P S$ & $P S$ & $P M$ & $P M$ & $P B$ & $P B$ & $P B$ \\
\hline$P M$ & $P M$ & $P M$ & $P M$ & $P B$ & $P B$ & $P B$ & $P B$ \\
\hline$P B$ & $P M$ & $P M$ & $P B$ & $P B$ & $P B$ & $P B$ & $P B$ \\
\hline
\end{tabular}




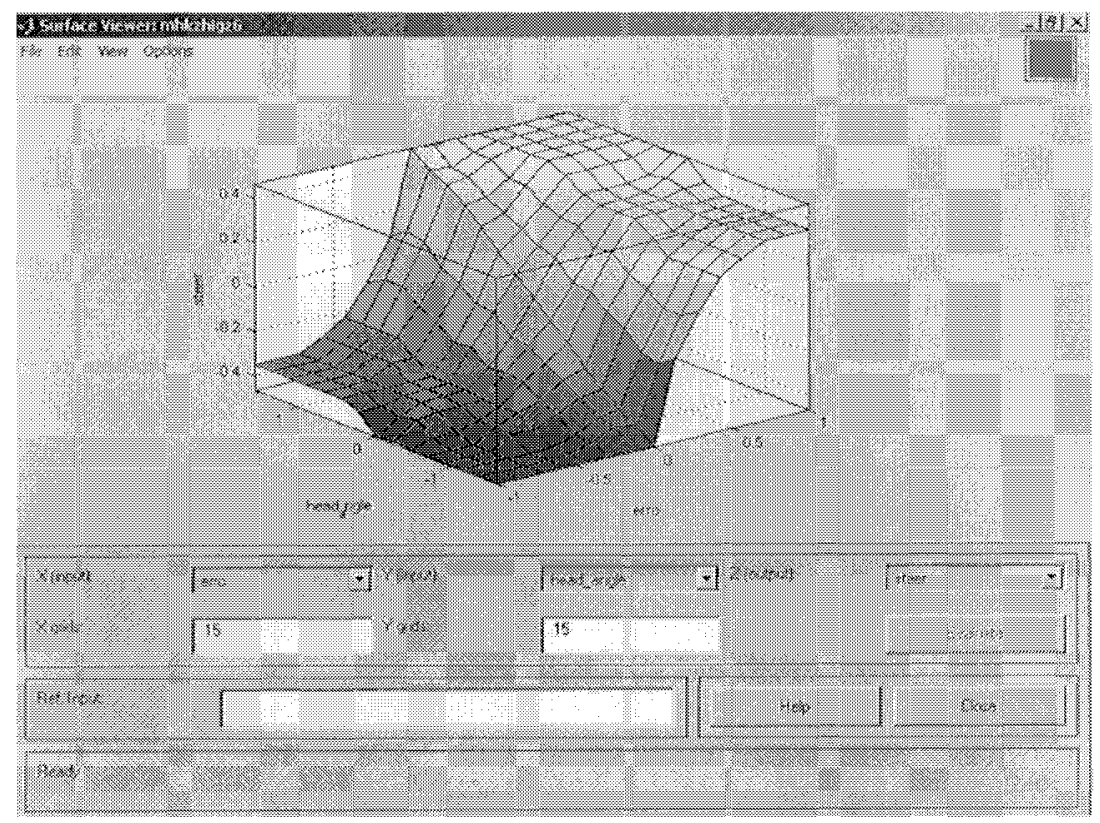

Figure3. Relation between input and output

\section{RESULTS AND DISCUSSION}

A tractor character parameter, such as $l_{1}=1.575 \mathrm{~m}, l_{2}=0.86 \mathrm{~m}$, $k_{1}=80 \mathrm{kN} / \mathrm{rad}, k_{2}=100 \mathrm{kN} / \mathrm{rad}, v=3 \mathrm{~m} / \mathrm{s}, M=4834 \mathrm{~kg} \cdot \mathrm{m}^{2}$. Firstly, the tractor dynamics model is founded, parameter of PID control is estimated by simulink, $k_{p}=10, k_{I}=1, k_{D}=5$; then Complex fuzzy control system is set up according to configuration(fig2), valve of fuzzy control is: yaw error is 0.2 and yaw angle is 0.157; when yaw error and yaw angle are more than valve, fuzzy control and PID will work together, which will enhance output but it can not exceed max output $( \pm 0.5233 \mathrm{rad})$; when yaw error or yaw angle is less than valve, fuzzy control will be cut down, and then, PID work by oneself. Since at this time tractor has been running along expectation route, it is viable that PID control yaw error alone. Original yaw error is 0 , finally yaw is 1 , and original yaw angle is 0 , in order that change route of tractor is simulated. Results are shown in figure 4 , and the results that fuzzy control working alone is shown in figure 5. The simulation results indicate that representation of complex fuzzy control is better than single fuzzy control, and embodiment driver knowledge which is suitable for tractor automatic steering system. 


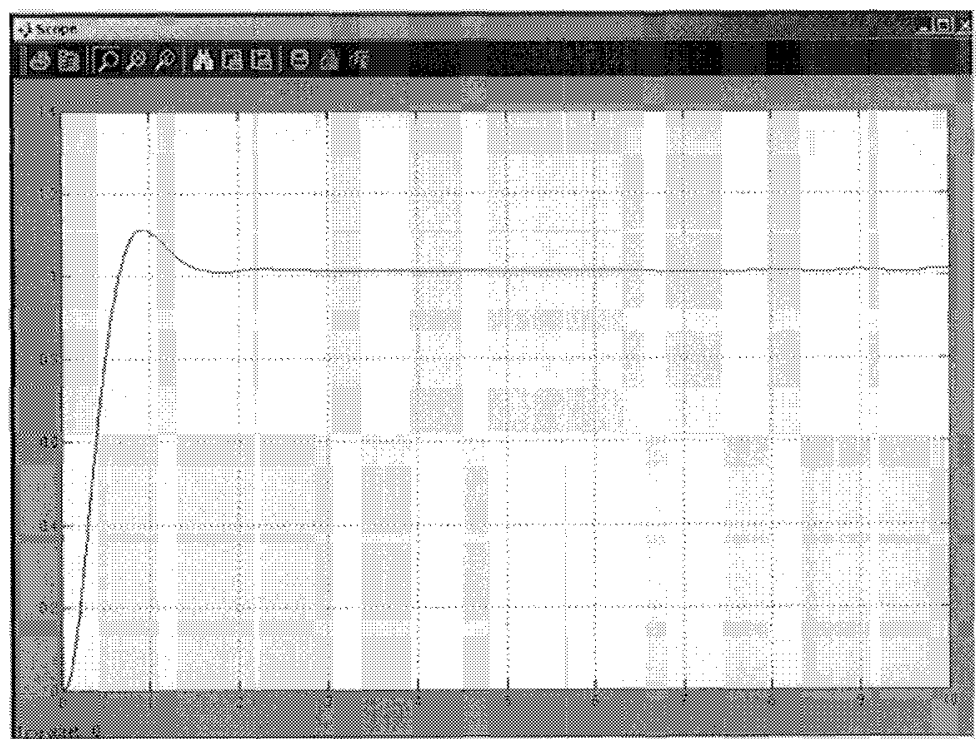

Figure4. Results of complex fuzzy control

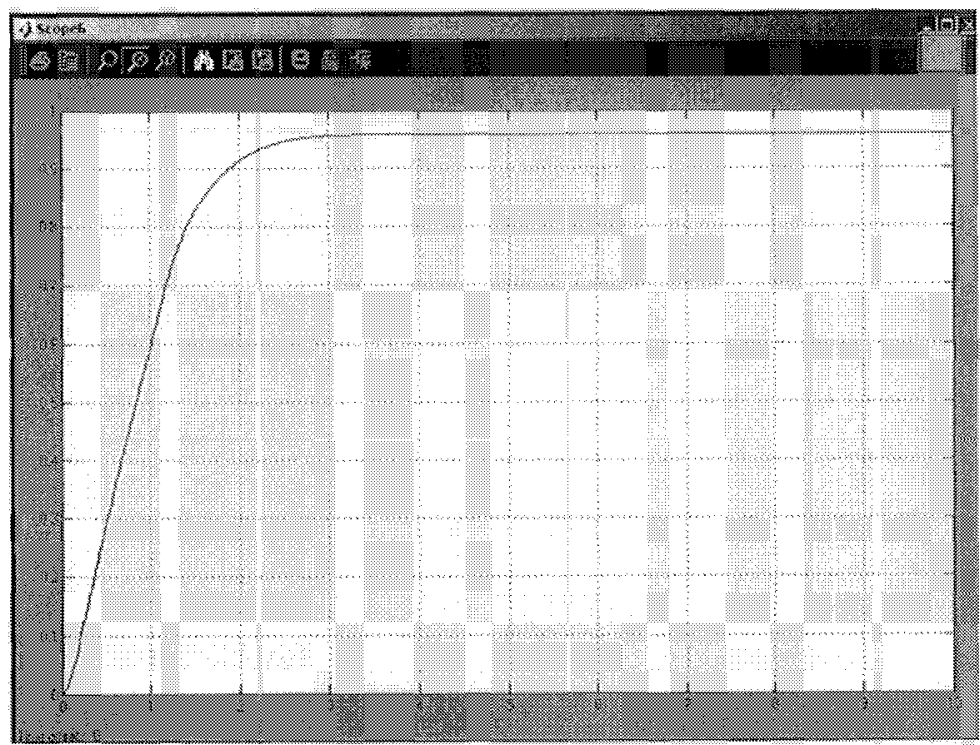

Figure 5. Results of fuzzy control

\section{CONCLUSIONS}

The main goal of this study is to develop a new method that can enhance navigation capable of autonomous tractor. Such a complex fuzzy control is 
put forward based on fuzzy control and PID, and tractor dynamics model is set up, which is base for simulation. Computer simulation analyses demonstrate that the complex fuzzy control can enhance control performance, such as reducing stabilization error and improving response speed besides realizing personification drive.

\section{REFERENCE}

[1] Chen tan. Cultivation manner changed by autonomous driving tractor. Science development and prospect, 1997, (3)

[2]Noboru Noguch, John F.Reid, Qin Zhang et al. Development of robot tractor based on RTK-GPS and gyroscope.ASAE paper:01-1195

[3]Simon Blackmore, Hans Werner Griepentrog, Henning Nielsen et al. Development of a deterministic autonomous tractor . Beijing : 2004 CIGR International Conference-Beijing, 11-14 October 2004

[4] Hans-peter Willumeit. Vehicle dynamics simulation and method[M]. Beijing: Beijing science and engineering university. 1998

[5] Yi jikai, hou yuanbin. Intelligent control technology[M]. Beijing: Beijing industry university. 2002.

[6] Wu xiaoli, lin zhehui. Matlab assistant fuzzy control[M]. Xi'an: Xi'an electron science and technology university, 2001 